\title{
Magnon-mediated binding between holes in an antiferromagnet
}

\author{
C. Brügger ${ }^{1}$, F. Kämpfer ${ }^{1, a}$, M. Pepe ${ }^{2}$, and U.-J. Wiese ${ }^{1}$ \\ 1 Institute for Theoretical Physics, Bern University, Sidlerstrasse 5, 3012 Bern, Switzerland \\ 2 Istituto Nazionale di Fisica Nucleare and Dipartimento di Fisica, Università di Milano-Bicocca, 3 Piazza della Scienza, \\ 20126 Milano, Italy
}

Received 8 June 2006 / Received in final form 3 October 2006

Published online 1st November 2006 - (c) EDP Sciences, Società Italiana di Fisica, Springer-Verlag 2006

\begin{abstract}
The long-range forces between holes in an antiferromagnet are due to magnon exchange. The one-magnon exchange potential between two holes is proportional to $\cos (2 \varphi) / \boldsymbol{r}^{2}$ where $\boldsymbol{r}$ is the distance vector of the holes and $\varphi$ is the angle between $\boldsymbol{r}$ and an axis of the square crystal lattice. One-magnon exchange leads to bound states of holes with antiparallel spins resembling $d$-wave symmetry.
\end{abstract}

PACS. 12.39.Fe Chiral Lagrangians - 74.20.Mn Nonconventional mechanisms (spin fluctuations, polarons and bipolarons, resonating valence bond model, anyon mechanism, marginal Fermi liquid, Luttinger liquid, etc.) - 75.30.Ds Spin waves - 75.50.Ee Antiferromagnetics

Over the past twenty years, understanding the dynamical mechanism responsible for high-temperature superconductivity [1] has remained a great challenge in condensed matter physics. Unfortunately, microscopic systems such as the Hubbard or $t-J$ model, which may indeed contain the relevant physics, have thus far neither been solved analytically nor numerically beyond half-filling. While analytic solutions usually suffer from uncontrolled approximations, numerical simulations suffer from the fermion sign problem. Although there have been numerous attempts to understand high-temperature superconductors via their undoped antiferromagnetic precursors [2-12], the dynamical role of spin fluctuations remains a controversial issue. In particular, there seems to be no agreement if two holes doped into an antiferromagnet can form a bound state or not. In the following we will use a systematic low-energy effective field theory for magnons and holes to show that - by one-magnon exchange - two holes can indeed form an infinite number of bound states. While it remains to be seen if this is directly relevant to high-temperature superconductivity, our results shed light on the mechanism responsible for the formation of charge pairs in the antiferromagnetic phase.

The low-energy physics of antiferromagnets is governed by magnons [13-15]. In analogy to chiral perturbation theory [16] — the effective theory for the pions of the strong interactions [17] — a systematic magnon effective theory was constructed in $[18,19]$. There have been a number of approaches $[7,9,10,20-22]$ that address the physics of both magnons and holes using effective field the-

\footnotetext{
a e-mail: fkampfer@itp.unibe.ch
}

ories. However, the different approaches do not agree even on basic issues like the fermion field content of the effective theory or on how various symmetries are realized on those fields. The effective field theory constructed here is the condensed matter analog of baryon chiral perturbation theory - the effective theory for pions and nucleons [23$26]$. It is based on a detailed symmetry analysis and uses a nonlinear realization of the spontaneously broken $S U(2)_{s}$ symmetry [27]. Analogies between pion and magnon dynamics have been investigated in [28]. While several elements of our effective theory have been used before, it differs from previous approaches in important respects. Just like chiral perturbation theory, our effective theory is based on a systematic low-energy expansion, which provides quantitatively correct predictions for a wide variety of microscopic systems including, e.g., the $t-J$ model. The effective theory itself is model-independent. Materialspecific properties enter the effective theory through a priori undetermined low-energy parameters, such as the spin stiffness or the spinwave velocity. The values of the lowenergy parameters for a concrete underlying microscopic system can be determined by comparison with experiments or with numerical simulations. Precise numerical simulations of low-energy observables, which are presently in progress in the $t-J$ model, constitute a most stringent test of the effective theory.

Magnon-mediated binding between pairs of holes has been studied before, most notably in the interesting work of Kuchiev and Sushkov [29]. Based on the microscopic $t-J$ model, these authors have derived the magnon-mediated forces between two holes residing in the same hole 
pocket and have solved the resulting Schrödinger equation. Here, for the first time, we derive the one-magnon exchange potential between holes in different hole pockets. Remarkably, in this case the corresponding Schrödinger equation can be solved completely analytically. The results of [29] are similar to ours in many respects. For example, the distance-dependence of the magnon-mediated forces is the same in both cases, and consequently both energy spectra contain infinitely many bound states. The effective field theory derivation of these results is conceptually particularly clean and transparent, and provides results that can be extended order by order in a systematic low-energy expansion.

The magnon field can be represented by a $\mathbb{C} P(1)$ projection matrix

$$
P(x)=\frac{1}{2}[\mathbb{1}+\boldsymbol{e}(x) \cdot \boldsymbol{\sigma}],
$$

that obeys $P(x)^{\dagger}=P(x), \operatorname{Tr} P(x)=1$, and $P(x)^{2}=P(x)$. Here $x=(\boldsymbol{x}, t)$ is a point in $(2+1)$-d space-time,

$$
\boldsymbol{e}(x)=(\sin \theta(x) \cos \varphi(x), \sin \theta(x) \sin \varphi(x), \cos \theta(x))
$$

is the staggered magnetization, and $\boldsymbol{\sigma}$ are the Pauli matrices. Under global spin rotations $g \in S U(2)_{s}$ the magnon field transforms as

$$
P(x)^{\prime}=g P(x) g^{\dagger},
$$

while under the displacement $D_{i}$ by one lattice spacing in the $i$-direction (which changes the sign of the staggered magnetization) it transforms as

$$
{ }^{D_{i}} P(x)=\mathbb{1}-P(x) .
$$

Under spatial rotations $O$, under reflections $R$, and under time-reversal $T$ one obtains

$$
\begin{array}{ll}
{ }^{O} P(x)=P(O x), & O x=\left(-x_{2}, x_{1}, t\right), \\
{ }^{R} P(x)=P(R x), & R x=\left(x_{1},-x_{2}, t\right), \\
{ }^{T} P(x)={ }^{D_{i}} P(T x), & T x=\left(x_{1}, x_{2},-t\right) .
\end{array}
$$

In order to couple holes to the magnons, a nonlinear realization of the spontaneously broken $S U(2)_{s}$ symmetry has been constructed [27]. It then manifests itself as a local symmetry in the unbroken $U(1)_{s}$ subgroup of $S U(2)_{s}$. The local $U(1)_{s}$ transformations are constructed from the global transformation $g \in S U(2)_{s}$ as well as from the local magnon field $P(x)$. First, one diagonalizes $P(x)$ by a unitary transformation $u(x) \in S U(2)_{s}$

$$
u(x) P(x) u(x)^{\dagger}=\frac{1}{2}\left[\mathbb{1}+\sigma_{3}\right], u_{11}(x) \geq 0 .
$$

Under a global $S U(2)_{s}$ transformation $g$ the diagonalizing field $u(x)$ transforms as

$$
u(x)^{\prime}=h(x) u(x) g^{\dagger},
$$

which defines the nonlinear symmetry transformation

$$
h(x)=\exp \left(i \alpha(x) \sigma_{3}\right) \in U(1)_{s} .
$$

Under the displacement symmetry $D_{i}$ one obtains

$$
{ }^{D_{i}} u(x)=\tau(x) u(x)
$$

with

$$
\tau(x)=\left(\begin{array}{cc}
0 & -\exp (-i \varphi(x)) \\
\exp (i \varphi(x)) & 0
\end{array}\right) .
$$

Next one constructs the anti-Hermitean composite field

$$
v_{\mu}(x)=u(x) \partial_{\mu} u(x)^{\dagger}=i\left(\begin{array}{cc}
v_{\mu}^{3}(x) & v_{\mu}^{+}(x) \\
v_{\mu}^{-}(x) & -v_{\mu}^{3}(x)
\end{array}\right),
$$

which transforms under $S U(2)_{s}$ as

$$
v_{\mu}(x)^{\prime}=h(x)\left[v_{\mu}(x)+\partial_{\mu}\right] h(x)^{\dagger} .
$$

The Abelian component $v_{\mu}^{3}(x)$ transforms like a $U(1)_{s}$ gauge field, while $v_{\mu}^{ \pm}(x)$ represent "charged" vector fields. Under the displacement symmetry $D_{i}$ the composite vector field transforms as

$$
{ }^{D^{i}} v_{\mu}(x)=\tau(x)\left[v_{\mu}(x)+\partial_{\mu}\right] \tau(x)^{\dagger},
$$

while under time-reversal

$$
{ }^{T} v_{i}(x)={ }^{D_{i}} v_{i}(T x), \quad{ }^{T} v_{t}(x)=-{ }^{D^{i}} v_{t}(T x) .
$$

Let us consider an effective theory with holes as the only charge carriers. In [27] we have considered charge carriers located near momenta $(0,0)$ and $\left(\frac{\pi}{a}, \frac{\pi}{a}\right)$ in the Brillouin zone (where $a$ is the lattice spacing). Here we consider hole pockets centered at

$$
k^{\alpha}=\left(\frac{\pi}{2 a}, \frac{\pi}{2 a}\right), \quad k^{\beta}=\left(\frac{\pi}{2 a},-\frac{\pi}{2 a}\right),
$$

which have been observed in ARPES measurements $[30-33]$ as well as in theoretical calculations in the $t-J$ model $[6,34,35]$. The hole fields $\psi_{s}^{f}(x)$ carry a "flavor" index $f=\alpha, \beta$ that characterizes the corresponding hole pocket. The index $s= \pm$ denotes spin parallel $(+)$ or antiparallel $(-)$ to the local staggered magnetization. Under the various symmetry operations the hole fields transform as

$$
\begin{aligned}
S U(2)_{s}: & \psi_{ \pm}^{f}(x)^{\prime} & =\exp ( \pm i \alpha(x)) \psi_{ \pm}^{f}(x), \\
U(1)_{Q}: & { }^{Q} \psi_{ \pm}^{f}(x) & =\exp (i \omega) \psi_{ \pm}^{f}(x), \\
D_{i}: & { }^{D}{ }^{i} \psi_{ \pm}^{f}(x) & =\mp \exp \left(i k_{i}^{f} a\right) \exp (\mp i \varphi(x)) \psi_{\mp}^{f}(x), \\
O: & { }^{O} \psi_{ \pm}^{\alpha}(x) & =\mp \psi_{ \pm}^{\beta}(O x),{ }^{O} \psi_{ \pm}^{\beta}(x)=\psi_{ \pm}^{\alpha}(O x), \\
R: & { }^{R} \psi_{ \pm}^{\alpha}(x) & =\psi_{ \pm}^{\beta}(R x),{ }^{R} \psi_{ \pm}^{\beta}(x)=\psi_{ \pm}^{\alpha}(R x), \\
T: & { }^{T} \psi_{ \pm}^{f}(x) & =\mp \exp (\mp i \varphi(T x)) \psi_{ \pm}^{f \dagger}(T x), \\
& { }^{T} \psi_{ \pm}^{f \dagger}(x) & = \pm \exp ( \pm i \varphi(T x)) \psi_{ \pm}^{f}(T x) .
\end{aligned}
$$

Here $\omega$ determines the $U(1)_{Q}$ fermion number transformation. Interestingly, in the effective theory the location $\left(k_{1}^{f}, k_{2}^{f}\right)$ of the hole pockets in the Brillouin zone of the underlying crystal lattice manifests itself through charges 
$k_{i}^{f} a= \pm \frac{\pi}{2}$ of an internal Abelian symmetry $D_{i}$. Defining a $U(1)_{s}$ covariant derivative

$$
D_{\mu} \psi_{ \pm}^{f}(x)=\left[\partial_{\mu} \pm i v_{\mu}^{3}(x)\right] \psi_{ \pm}^{f}(x),
$$

the leading terms in the effective Lagrangian are

$$
\begin{aligned}
& \mathcal{L}\left[\psi_{s}^{f \dagger}, \psi_{s}^{f}, P\right]=\rho_{s} \operatorname{Tr}\left[\partial_{i} P \partial_{i} P+\frac{1}{c^{2}} \partial_{t} P \partial_{t} P\right] \\
& +\sum_{f=\alpha, \beta ; s=+,-}\left[M \psi_{s}^{f \dagger} \psi_{s}^{f}+\psi_{s}^{f \dagger} D_{t} \psi_{s}^{f}+\frac{1}{2 M^{\prime}} D_{i} \psi_{s}^{f \dagger} D_{i} \psi_{s}^{f}\right. \\
& +\sigma_{f} \frac{1}{2 M^{\prime \prime}}\left(D_{1} \psi_{s}^{f \dagger} D_{2} \psi_{s}^{f}+D_{2} \psi_{s}^{f \dagger} D_{1} \psi_{s}^{f}\right) \\
& +\Lambda\left(\psi_{s}^{f \dagger} v_{1}^{s} \psi_{-s}^{f}+\sigma_{f} \psi_{s}^{f \dagger} v_{2}^{s} \psi_{-s}^{f}\right)+N_{1} \psi_{s}^{f \dagger} v_{i}^{s} v_{i}^{-s} \psi_{s}^{f} \\
& \left.+\sigma_{f} N_{2}\left(\psi_{s}^{f \dagger} v_{1}^{s} v_{2}^{-s} \psi_{s}^{f}+\psi_{s}^{f \dagger} v_{2}^{s} v_{1}^{-s} \psi_{s}^{f}\right)+\frac{G_{1}}{2} \psi_{s}^{f \dagger} \psi_{s}^{f} \psi_{-s}^{f \dagger} \psi_{-s}^{f}\right] \\
& +\sum_{s=+,-}\left[G_{2} \psi_{s}^{\alpha \dagger} \psi_{s}^{\alpha} \psi_{s}^{\beta \dagger} \psi_{s}^{\beta}+G_{3} \psi_{s}^{\alpha \dagger} \psi_{s}^{\alpha} \psi_{-s}^{\beta \dagger} \psi_{-s}^{\beta}\right] .
\end{aligned}
$$

Here $\rho_{s}$ is the spin stiffness, $c$ is the spinwave velocity, $M, M^{\prime}$ and $M^{\prime \prime}$ are the rest mass and kinetic masses of a hole, $\Lambda$ is a hole-one-magnon, and $N_{1}$ and $N_{2}$ are holetwo-magnon couplings, and $G_{1}, G_{2}$, and $G_{3}$ are 4 -fermion contact interactions. All these low-energy parameters take real values. The sign $\sigma_{f}$ is + for $f=\alpha$ and - for $f=$ $\beta$. Interestingly, the above Lagrangian has an accidental global $U(1)_{F}$ flavor symmetry that acts as

$$
U(1)_{F}:{ }^{F} \psi_{ \pm}^{f}(x)=\exp \left(\sigma_{f} i \eta\right) \psi_{ \pm}^{f}(x) .
$$

In addition, for $c \rightarrow \infty$ it also has an accidental Galilean symmetry. Both accidental symmetries are explicitly broken at higher orders of the derivative expansion.

Our treatment of the forces between two holes is analogous to the effective theory for light nuclei [36-39] in which one-pion exchange dominates the long-range forces. We now calculate the one-magnon exchange potential. For this purpose, we expand in the magnon fluctuations $m_{1}(x)$, $m_{2}(x)$ around the ordered staggered magnetization, i.e.

$$
\begin{aligned}
\boldsymbol{e}(x) & =\left(\frac{m_{1}(x)}{\sqrt{\rho_{s}}}, \frac{m_{2}(x)}{\sqrt{\rho_{s}}}, 1\right)+\mathcal{O}\left[m^{2}\right] \Rightarrow v_{\mu}^{3}(x)=\mathcal{O}\left[m^{2}\right], \\
v_{\mu}^{ \pm}(x) & =\frac{1}{2 \sqrt{\rho_{s}}} \partial_{\mu}\left[m_{2}(x) \pm i m_{1}(x)\right]+\mathcal{O}\left[m^{2}\right] .
\end{aligned}
$$

Since vertices with $v_{\mu}^{3}(x)$ (contained in $D_{\mu}$ ) involve at least two magnons, one-magnon exchange results from vertices with $v_{\mu}^{ \pm}(x)$ only. As a consequence, two holes can exchange a single magnon only if they have antiparallel spins $(+$ and -$)$, which are both flipped in the magnon exchange process. It is straightforward to evaluate the Feynman diagram describing one-magnon exchange shown in Figure 1.

In coordinate space the resulting potentials for the various combinations of flavors take the form

$$
V^{f f}(\boldsymbol{r})=\sigma_{f} \gamma \frac{\sin (2 \varphi)}{\boldsymbol{r}^{2}}, V^{\alpha \beta}(\boldsymbol{r})=\gamma \frac{\cos (2 \varphi)}{\boldsymbol{r}^{2}},
$$

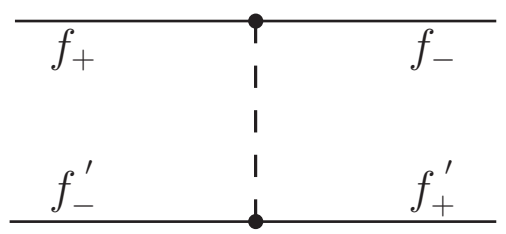

Fig. 1. Feynman diagram for one-magnon exchange between two holes with antiparallel spins undergoing a spin-flip.

with $\gamma=\Lambda^{2} /\left(2 \pi \rho_{s}\right)$. Here $\boldsymbol{r}$ is the distance vector between the two holes and $\varphi$ is the angle between $\boldsymbol{r}$ and the $x$-axis. It should be noted that the one-magnon exchange potential is instantaneous although magnons travel with the finite speed $c$. Retardation effects occur only at higher orders.

Next we study the Schrödinger equation for the relative motion of two holes with flavors $\alpha$ and $\beta$

$$
\left(\begin{array}{cc}
-\frac{1}{M^{\prime}} \Delta & V^{\alpha \beta}(\boldsymbol{r}) \\
V^{\alpha \beta}(\boldsymbol{r}) & -\frac{1}{M^{\prime}} \Delta
\end{array}\right)\left(\begin{array}{l}
\Psi_{1}(\boldsymbol{r}) \\
\Psi_{2}(\boldsymbol{r})
\end{array}\right)=E\left(\begin{array}{l}
\Psi_{1}(\boldsymbol{r}) \\
\Psi_{2}(\boldsymbol{r})
\end{array}\right) .
$$

The components $\Psi_{1}(\boldsymbol{r})$ and $\Psi_{2}(\boldsymbol{r})$ are probability amplitudes for the spin-flavor combinations $\alpha_{+} \beta_{-}$and $\alpha_{-} \beta_{+}$, respectively. The potential $V^{\alpha \beta}(\boldsymbol{r})$ couples the two channels because magnon exchange is accompanied by a spin-flip. The above Schrödinger equation does not yet account for the short-distance forces arising from 4 -fermion contact interactions. Their effect will be incorporated later by a boundary condition on the wave function near the origin. Making the ansatz

$$
\Psi_{1}(\boldsymbol{r}) \pm \Psi_{2}(\boldsymbol{r})=R(r) \chi_{ \pm}(\varphi)
$$

for the angular part of the wave function one obtains

$$
-\frac{d^{2} \chi_{ \pm}(\varphi)}{d \varphi^{2}} \pm M^{\prime} \gamma \cos (2 \varphi) \chi_{ \pm}(\varphi)=-\lambda \chi_{ \pm}(\varphi)
$$

The solutions of this Mathieu equation with the lowest eigenvalue $\lambda$ (given here only to the leading order $\gamma^{2}$ ) is

$$
\chi_{ \pm}(\varphi)=\frac{1}{\sqrt{\pi}} \operatorname{ce}_{0}\left(\varphi, \pm \frac{1}{2} M^{\prime} \gamma\right), \lambda=\frac{1}{8}\left(M^{\prime} \gamma\right)^{2} .
$$

The periodic Mathieu function $\operatorname{ce}_{0}\left(\varphi, \frac{1}{2} M^{\prime} \gamma\right)$ [40] is illustrated for $M^{\prime} \gamma=2.5$ in Figure 2.

The radial Schrödinger equation takes the form

$$
-\left[\frac{d^{2} R(r)}{d r^{2}}+\frac{1}{r} \frac{d R(r)}{d r}\right]-\frac{\lambda}{r^{2}} R(r)=M^{\prime} E R(r) .
$$

As it stands, the above equation is ill-defined because an attractive $\frac{1}{r^{2}}$ potential is too singular at the origin. However, one should keep in mind that we have not yet incorporated the short-range contact interactions. A consistent description of the short-distance physics requires ultraviolet regularization and subsequent renormalization of the Schrödinger equation as discussed in [41]. Instead of proceeding systematically in this way, here we model the short-distance repulsion between two holes by a hard 


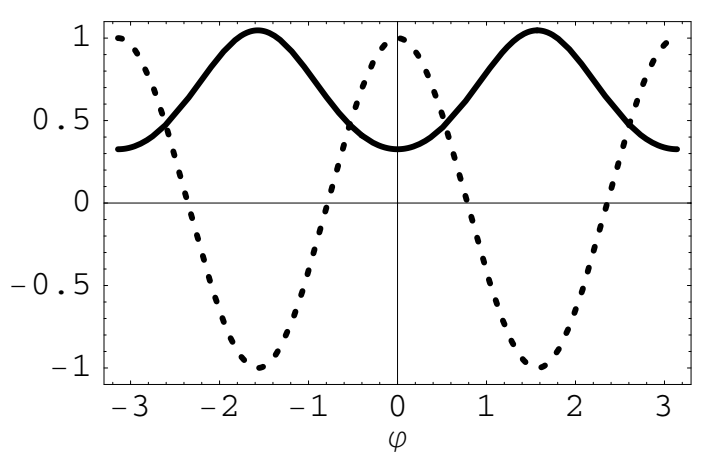

Fig. 2. Angular wave function $\operatorname{ce}_{0}\left(\varphi, \frac{1}{2} M^{\prime} \gamma\right)$ (solid curve) and angle-dependence $\cos (2 \varphi)$ of the potential (dotted curve).

core of radius $r_{0}$, i.e. we require $R\left(r_{0}\right)=0$. The radial Schrödinger equation for the bound states is solved by Bessel functions

$$
R(r)=A K_{\nu}\left(\sqrt{M^{\prime}\left|E_{n}\right|} r\right), \nu=i \sqrt{\lambda}
$$

The energy (determined from $K_{\nu}\left(\sqrt{M^{\prime}\left|E_{n}\right|} r_{0}\right)=0$ ) is given by

$$
E_{n} \sim-\left(M^{\prime} r_{0}^{2}\right)^{-1} \exp (-2 \pi n / \sqrt{\lambda})
$$

for large $n$. While the highly excited states have exponentially small energy and exponentially large size, for sufficiently small $r_{0}$ or sufficiently large coupling $\Lambda$ the ground state is strongly bound. It should be noted that the wave functions with angular part $\chi_{+}(\varphi)$ and $\chi_{-}(\varphi)$ have the same energy, i.e. the states are two-fold degenerate. Combining the two degenerate ground states to eigenstates of the rotation $O$ one obtains the probability distribution illustrated in Figure 3, which resembles $d$-wave symmetry. It should, however, be noted that, due to the nontrivial rotation properties of flavor, the wave function is suppressed - but not equal to zero - along the lattice diagonals. Pairs of holes with equal flavor can also form. The corresponding wave functions will be discussed elsewhere. Whether pairs of the same or of different flavors are more strongly bound depends on the values of the low-energy parameters. Here we have concentrated on $\alpha \beta$ pairs because they have important properties observed in the cuprates.

Since we have now established that two holes in an antiferromagnet can form a bound state by exchanging magnons, it is natural to ask if and how this may be related to high-temperature superconductivity. Quantitatively these questions will be addressed elsewhere. Here we argue just qualitatively. If pairs of holes form bound states, at a sufficiently low temperature $T_{c}$ these pairs will condense, thus leading to Bose-Einstein condensation or to magnon-mediated superconductivity. Here we do not attempt to estimate $T_{c}$, because this involves a delicate interplay between long- and short-range interactions. Instead we concentrate on the universal aspects of the dynamics resulting from the long-range magnon-mediated forces only. First, one-magnon exchange only binds holes with antiparallel spins, and indeed the Cooper pairs in a

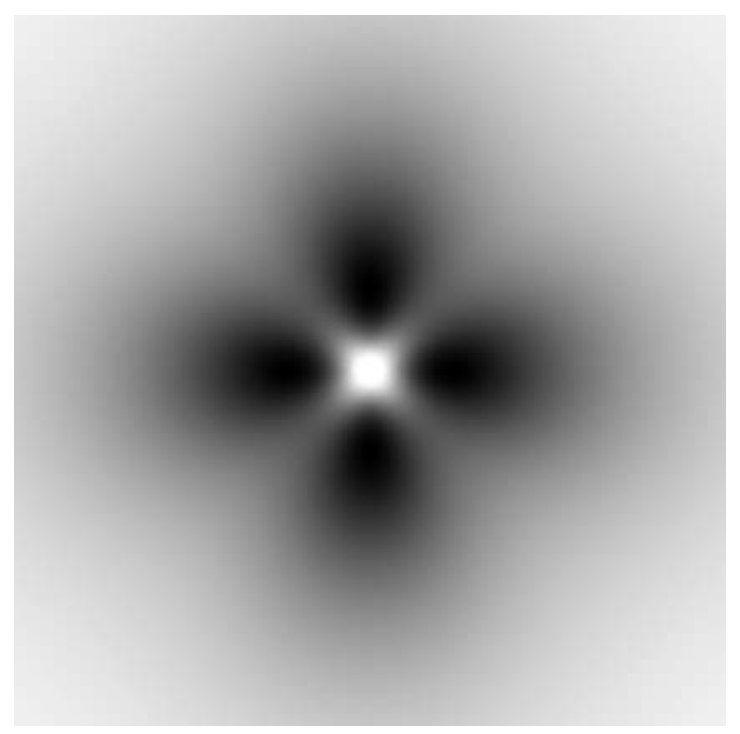

Fig. 3. Probability distribution for the ground state of two holes with flavors $\alpha$ and $\beta$.

high-temperature superconductor are spin singlets. Second, the characteristic angular dependence $\cos (2 \varphi)$ of the one-magnon exchange potential leads to the peculiar $\operatorname{ce}_{0}\left(\varphi, \frac{1}{2} M^{\prime} \gamma\right)$ orbital structures of the hole pair wave function which yields the $d$-wave characteristics observed in the cuprates.

Besides basic principles of quantum field theory, such as locality and unitarity, the effective theory of magnons and holes relies only on a few experimentally well verified dynamical assumptions - most important the spontaneous breaking of the $S U(2)_{s}$ spin symmetry down to $U(1)_{s}$ and the location of hole pockets at $\left(\frac{\pi}{2 a}, \pm \frac{\pi}{2 a}\right)$. It is remarkable that the existence of bound states between holes in an antiferromagnet can be inferred from so little input. The effective theory provides the detailed analytic form of the wave function for a pair of holes with different flavors $\alpha \beta$. While the corresponding probability distribution resembles $d$-wave characteristics, due to the nontrivial flavor structure the rotation symmetry $O$ is, nevertheless, realized in a more complicated way.

It is natural to ask if the effective theory can be applied to the high-temperature superconductors themselves. Since this theory relies on the spontaneous breakdown of the $S U(2)_{s}$ symmetry, and since high-temperature superconductivity arises only after antiferromagnetism has been destroyed, this may seem doubtful. However, while the perturbative treatment of the effective theory breaks down in the superconducting phase, the effective theory itself does not, as long as spin fluctuations remain among the relevant low-energy degrees of freedom. While it remains to be seen if nonperturbative investigations of the effective theory can shed light on the phenomenon of hightemperature superconductivity itself, it seems clear already that the systematic low-energy effective field theory approach to the dynamics of charge carriers in antiferromagnets is promising. 
We are indebted to J. Gasser for illuminating discussions. This work was supported in part by the Schweizerischer Nationalfonds.

\section{References}

1. J.C. Bednorz, K.A. Müller, Z. Phys. B 64, 189 (1986)

2. K. Miyake, S. Schmitt-Rink, C.M. Varma, Phys. Rev. B 34, R6554 (1986)

3. D.J. Scalapino, E.J. Loh, J.E. Hirsch, Phys. Rev. B 34, $8190(1986)$

4. P.W. Anderson, Science 235, 1196 (1987)

5. C. Gros, R. Joynt, T.M. Rice, Phys. Rev. B 36, 381 (1987)

6. S.A. Trugman, Phys. Rev. B 37, 1597 (1988)

7. B.I. Shraiman, E.D. Siggia, Phys. Rev. Lett. 60, 740 (1988); B.I. Shraiman, E.D. Siggia, Phys. Rev. Lett. 61, 467 (1988); B.I. Shraiman, E.D. Siggia, Phys. Rev. Lett. 62, 1564 (1989); B.I. Shraiman, E.D. Siggia, Phys. Rev. B 46, 8305 (1992)

8. J.R. Schrieffer, X.G. Wen, S.C. Zhang, Phys. Rev. Lett. 60, 944 (1988); Phys. Rev. B 39, 11663 (1989)

9. X.G. Wen, Phys. Rev. B 39, R7223 (1989)

10. R. Shankar, Phys. Rev. Lett. 63, 203 (1989); R. Shankar, Nucl. Phys. B 330, 433 (1990)

11. P. Monthoux, A.V. Balatsky, D. Pines, Phys. Rev. Lett. 67, 3448 (1991); P. Monthoux, A.V. Balatsky, D. Pines, Phys. Rev. B 46, 14803 (1992)

12. D. Poilblanc, J. Riera, E. Dagotto, Phys. Rev. B 49 R12318 (1994)

13. S. Chakravarty, B.I. Halperin, D.R. Nelson, Phys. Rev. B 39, 2344 (1989)

14. H. Neuberger, T. Ziman, Phys. Rev. B 39, 2608 (1989)

15. D.S. Fisher, Phys. Rev. B 39, 11783 (1989)

16. J. Gasser, H. Leutwyler, Nucl. Phys. B 250, 465 (1985)

17. S. Weinberg, Physica A 96, 327 (1979)

18. P. Hasenfratz, H. Leutwyler, Nucl. Phys. B 343, 241 (1990)

19. P. Hasenfratz, F. Niedermayer, Z. Phys. B 92, 91 (1993)
20. H.J. Schulz, Phys. Rev. Lett. 65, 2462 (1990)

21. Z.Y. Weng, C.S. Ting, T.K. Lee, Phys. Rev. B 43, 3790 (1991)

22. C. Kübert, A. Muramatsu, Phys. Rev. B 47, 787 (1993); e-print arXiv: cond-mat/9505105

23. J. Gasser, M.E. Sainio, A. Svarc, Nucl. Phys. B 307, 779 (1988)

24. E. Jenkins, A. Manohar, Phys. Lett. B 255, 558 (1991)

25. V. Bernard et al., Nucl. Phys. B 388, 315 (1992)

26. T. Becher, H. Leutwyler, Eur. Phys. J. C 9, 643 (1999)

27. F. Kämpfer, M. Moser, U.-J. Wiese, Nucl. Phys. B 729, $317(2005)$

28. O. Bär, M. Imboden, U.-J. Wiese, Nucl. Phys. B 686, 347 (2004)

29. M.Y. Kuchiev, O.P. Sushkov, Physica C 218, 197 (1993)

30. B.O. Wells et al., Phys. Rev. Lett. 74, 964 (1995)

31. S. La Rosa et al., Phys. Rev. B 56, R525 (1997)

32. C. Kim et al., Phys. Rev. Lett. 80, 4245 (1998)

33. F. Ronning et al., Science 282, 2067 (1998)

34. V. Elser, D.A. Huse, B.I. Shraiman, E.D. Siggia, Phys. Rev. B 41, 6715 (1990)

35. M. Brunner, F.F. Assaad, A. Muramatsu, Phys. Rev. B 62, 15480 (2000)

36. S. Weinberg, Phys. Lett. B 251, 288 (1990); S. Weinberg, Nucl. Phys. B 363, 3 (1991); S. Weinberg, Phys. Lett. B 295, 114 (1992)

37. D.B. Kaplan, M.J. Savage, M.B. Wise, Phys. Lett. B 424, 390 (1998); D.B. Kaplan, M.J. Savage, M.B. Wise, Nucl. Phys. B 534, 329 (1998)

38. E. Epelbaum, W. Glöckle, U.-G. Meissner, Nucl. Phys. A 637, 107 (1998); E. Epelbaum, W. Glöckle, U.-G. Meissner, Nucl. Phys. A 714, 535 (2003)

39. P.F. Bedaque, H.-W. Hammer, U. van Kolck, Phys. Rev. C 58, R641 (1998); P.F. Bedaque, H.-W. Hammer, U. van Kolck, Phys. Rev. Lett. 82, 463 (1999)

40. M. Abramowitz, I.A. Stegun, Handbook of Mathematical Functions (Dover Publications, Inc., New York, 1972)

41. G.P. Lepage, e-print arXiv:nucl-th/9706029 